\title{
Impact on Drug Costs and Utilization of a Clinical Pharmacist in a Multisite Primary Care Medical Group
}

\author{
SCOT WALKER, PharmD, MS, BCPS, and CHARLES W. WILLEY, MD
}

\begin{abstract}
OBJECTIVES: To measure the cost and utilization outcomes of a pharmacist intervention in a primary care medical group operating under a financial risk contract with a health plan.

METHODS: A prestudy-poststudy design using national drug utilization for the comparison was employed to assess the impact of physician-prescriber education using information derived from prescriber-specific drug cost and utilization analyses. Eight therapeutic areas involving 10 drug classes were targeted (antihistamines, nasal steroids, proton pump inhibitors, histamine-2 receptor antagonists, antidepressants, nonsteroidal anti-inflammatory drugs [NSAIDs, including celecoxib and rofecoxib], antibiotics, angiotensin-converting enzyme [ACE] inhibitors, calcium channel blockers, and statins). The subject medical group was an independent, nonacademic, ambulatory, primary care medical practice of 65 primary care physicians consisting of 50 internists, 14 pediatricians, and 1 family practitioner. Drug costs were measured as net medical group costs per enrolled member per year (PMPY), the product of the average cost per prescription, and the number of prescriptions PMPY.
\end{abstract}

RESULTS: The increase in drug cost PMPY for this medical group was $1.7 \%$ in a commercial contract in 1999 compared with an increase of $31.2 \%$ for health plans nationwide. Utilization as measured by prescriptions PMPY did not change in the national data comparison over the 2-year observation period and increased by $4.0 \%$ in the medical group. The national average cost per prescription claim increased $31.2 \%$ in 1999 compared with a decrease of $2.1 \%$ in the medical group. The decrease in average drug cost in this medical group was associated with an increase in the use of preferred drugs, including more generic drugs.

CONCLUSION: A targeted educational program for physician-prescribers conducted by a clinical pharmacist working for a primary care medical group can reduce the expenditures for outpatient drug therapy by lowering the average cost per pharmacy claim.

KEYWORDS: Drug costs, Utilization, Medical group, Physician education

J Manag Care Pharm. 2004;10(4):345-54
I $\mathrm{n}$ an effort to control rising medical costs, managed care organizations negotiate financial risk arrangements with physicians. Some risk contracts include only expenditures involved in office visits, procedures, and hospitalizations billed by the primary care physician while other risk contracts include a wider range of costs such as the drugs and charges generated by specialists. This second type of risk contract, commonly referred to as a global risk contract, is more challenging for a medical group to manage due to the large amount of data and analyses that are required to measure health care utilization and expenditures. ${ }^{1}$ This study examined one method employed by a community-based, independent primary care medical group to control drug expenditures for health plan members.

Nationally, several factors have led to an increase in drug expenditures. New drugs were introduced that are generally more expensive. Direct-to-consumer advertising contributed to patient demand for more expensive drugs. New indications for drugs already marketed also led to an increase in utilization. The prevalent beliefs that access to health care and drugs are an entitlement and that health care being mostly funded by employers, health plans, or the government has insulated consumers from rising health care costs has helped to increase utilization. ${ }^{2}$

Between 1997 and 1998, the per-member-per-year (PMPY) drug cost of Esse Health, a 65-physician, community based, independent, nonacademic, medical group located in St. Louis, increased $29.8 \%$ compared with the national rate of growth in drug spending of $10.6 \% .^{3}$ The medical group, operating from 24 clinical locations, had successfully managed office visits, procedures, referrals, and hospitalizations, but drug costs had increased at a rate perceived as uncontrolled. The medical group had accepted drug cost as part of the global risk contract with the health plan but did not have input into drug formulary decisions. The financial loss in the drug risk pool was adversely affecting the overall performance of the global contract and had the potential to cause the group to experience significant financial harm.

The global risk contract covered members enrolled in a commercial managed care plan offered by several local employers. The medical group was incorporated under a single tax ID with risk assigned to physicians according to the number of members who had enrolled in the plan. No other provider or provider group had a capitated contract in the commercial managed care plan. The number of members assigned to the study medical group was 14,005 in 1997, 12,128 in 1998, and 11,119 in 1999. There were no significant changes to the basic drug benefit design during the 2-year period (1998 and 1999). 


\section{FIGURE 1}

\section{Care Algorithms for}

Clinical Pharmacist Interventions

\begin{abstract}
Allergy: Nasal corticosteroids were recommended as initial treatment for allergic rhinitis. Chlorpheniramine or brompheniramine were recommended for patients who did not tolerate or refused intranasal treatment. Fexofenadine was recommended if a low-sedating antihistamine (LSA) was needed. Physicians were reminded to avoid using an LSA to treat rhinorrhea secondary to upper respiratory infection.
\end{abstract}

Nonulcerative dyspepsia (NUD) and gastroesophageal reflux disease (GERD): Physicians were requested to emphasize lifestyle changes (e.g., elevate the head of the bed, avoid alcohol before retiring and allow at least 2 hours between dinner and retiring) in all GERD patients and NUD patients with predominant GERD symptoms. Generic ranitidine was recommended as first line drug treatment in patients with mild disease. Lansoprazole was recommended for patients requiring a proton pump inhibitor.

Depression: Desipramine or trazodone were recommended as initial treatment for depression. Citalopram, sertraline, or paroxetine could be considered in patients requiring a selective seratonin reuptake inhibitor. In appropriate patients, a half-tablet strategy could be used with citalopram, sertraline or paroxetine. It was recommended that the physician review the medical chart to ensure that the patient had completed at least 9 months of treatment or required maintenance therapy.

Antibiotics: The medical staff was encouraged to decrease the use of antibiotics for upper respiratory infections URIs) that are predominantly viral. If the URI was felt to be bacterial, it was recommended that physicians use erythromycin, doxycycline, or amoxicillin as initial therapy. Newer antibiotics should be reserved for patients who are institutionalized, smoke, have chronic obstructive pulmonary disease, are immunocompromise, or in whom resistance is suspected. An educational handout for patients was developed to explain why antibiotics are not always needed. Physicians were encouraged to use the handout to decease patient expectations for an antibiotic.

Angiotensin-converting enzyme inhibitors (ACEIs): Generic captopril was recommended as initial therapy. If a once-daily drug was needed, it was suggested that physicians consider having patients pay cash and take a half tablet of moexipril, since the cost was less than the copay for a brand-name ACE inhibitor prescription (there were no generic ACE inhibitors at the time except captopril).

Calcium channel blockers (CCBs): The medical staff was asked to decrease use of dihydropyridine CCBs since, for most indications, beta-blockers and/or angiotensin-converting enzyme inhibitors have demonstrated a long-term benefit. If a dihydropyridine CCB was needed, physicians were advised to consider felodipine

Dyslipidemia and statin therapy: Physicians were advised to aggressively lower their patient's LDL to prevent future cardiovascular complications. Atorvastatin was recommended as the preferred statin and a half-tablet strategy could be considered in appropriate patients to reduce the average cost per day of therapy.

Nonsteroidal anti-inflammatory drugs (NSAIDs): It was recommended that at least 2 generic NSAIDs be tried before using a branded NSAID. COX-2 inhibitors are no better than NSAIDs in controlling pain and inflammation. Since a reduction in gastrointestinal (GI) bleeding is only theoretical, COX-2 inhibitors should be reserved for high-risk patients. Risk factors for GI bleeding include a prior history of a GI ulcer or bleed, patient aged $>60$ years, high-dose NSAID or aspirin, and concurrent use of corticosteroids or anticoagulants
Most members had a 2-tier copay benefit with a lower copayment for generic drugs and a higher copayment for brand-name formulary drugs. Fewer than $20 \%$ of members were enrolled in 3-tier copay drug benefit designs. The medical group did not assess if there were changes in the individual drug plans offered to the health plan members of the various employers.

The medical group examined the options for managing the growth in drug expenditures, including early termination of the 3 -year risk contract or requesting the educational assistance of the clinical and administrative staff of the health plan. Ultimately, the medical group elected a self-management option that involved creation of a data warehouse and use of these data in a prescriber education intervention.

The medical group hired a clinical pharmacist to analyze drug utilization and assist in the creation of education tools to use with physician-prescribers. Educational interventions with prescribers were designed at year-end 1998 to blunt the increase in drug costs incurred by health plan members assigned to the medical group. The medical group was interested in the analysis of the impact of the intervention on average cost per claim (prescription) and utilization.

\section{Methods}

A retrospective pretest and posttest design was used to compare the results of the medical group with national data on drug cost and utilization. During 1998, the pharmacist studied the communication infrastructure of the group and the lines of authority. An analysis of drug utilization was commenced. The interventions in physician education regarding observed versus desired prescribing patterns began in January 1999.

It was not possible to assess the education intervention in a prospective manner with a control group within the medical group because the medical group used electronic mail extensively for group discussion and held regular monthly meetings. It was also not possible to test the intervention against another large primary care practice in the region since there was not a comparable primary care group in the region with a global risk contract. The option for analysis was comparison with national composite data for the same time period. The Novartis Pharmacy Benefit Report, 1999 and 2000 editions, was chosen for benchmark comparative data. ${ }^{3,4}$

The medical group was composed of 65 physicians: 50 internists, 14 pediatricians, and 1 family practitioner, distributed among 24 practice sites. This mix of primary care physicians (77\% internists) resulted in a member population that had a higher proportion of older adults who would contribute to higher PMPY drug cost than national averages for primary care groups that contained a higher percentage of pediatricians and family practitioners. Drug utilization data were only available for patients in global risk contracts so a comparison to risk and nonrisk patients was not possible.

The 1999 and 2000 editions of the Novartis Pharmacy Benefit 
Report organized the data in different ways. In the 1999 edition, for claims incurred in 1998, drug utilization was organized by model type, ${ }^{3}$ while in the 2000 edition, for claims incurred in 1999, drug utilization was reported by line of business. ${ }^{4}$ For the purposes of comparison for this study, the overall trend in drug utilization was used, since these data were reported in a consistent manner for the 2 years. ${ }^{5}$ The national data included Medicare and Medicaid enrollees as well as commercial members in the national data, while only commercial members were enrolled in the medical group population that is the subject of this study.

It was felt that in order to change the prescribing behaviors of the physicians, an educational intervention was needed. The intervention would use marketing techniques to gain acceptance and participation by the medical staff. Soumerai has described an 8-step social marketing process to change physician behavior. ${ }^{6}$ In the first step, baseline information is collected to determine current practice and barriers to change. Following that, an educational message to a target group of physicians is developed. Special emphasis is placed on gaining acceptance of the opinion leaders of the group. The process also includes developing objectives, establishing credibility, and encouraging physician participation. Concise educational materials along with repeated key messages help to drive the primary points of the educational intervention. Finally, reinforcement of the key messages is provided with additional physician contact.

The intervention to reduce drug costs in the present study involved using a combination of drug utilization analysis, academic detailing, and staff education to influence prescribing. The methods of Soumerai were modified to take advantage of the unique dynamics of the group. Baseline data were determined through an analysis of the prescription claims database. Group meetings with physicians and participation by the clinical pharmacist in the group's utilization management committee helped to determine current practice and barriers to change. Eight therapeutic areas (involving 10 drug classes) were chosen for intervention, and care management recommendations were developed for each therapeutic area (Figure 1). The target and care recommendations were designed to decrease drug cost per claim (i.e., per day of therapy) while providing a similar or better clinical benefit. The medical group was divided into 4 geographic regions. Each region met on a weekly basis to discuss patients. The regional group leader and the most influential opinion maker in each of the 4 regions were recruited for acceptance of the interventions. This provided local idea champions to aid in modifying the behavior of the entire group.

Drug utilization reports that were prepared by the pharmacy benefit management (PBM) company employed by the health plan did not provide enough useful data to influence physician prescribing, because, for example, the reports were sorted by national drug code (NDC) number. This fine granularity of the data did not permit the presentation of summary data that would support physician education; e.g., a report from the PBM to a pediatrician might contain 7 categories for different formulations of amoxicillin.

In order to measure the utilization of health care resources in risk contracts and produce useful reports, the medical group created a relational database, which stored all medical and pharmacy claims obtained from the HMO. A full-time data analyst reviewed the data, resolved discrepancies with the HMO, and scrubbed the data for importation into the database. It was necessary to clean the claims data prepared by the health plan due to frequent problems such as miscoding. Correction of these claims was necessary for data integrity as well as to ensure assignment of the claims and costs to the correct risk pool. The data analyst spent approximately 5 hours per week qualifying the medical and drug claim data, and a database administrator spent approximately 5 hours per month formatting and adding the drug claim information into the data warehouse. The clinical pharmacist spent approximately 25 hours each week analyzing the data, creating reports for the medical staff, designing educational interventions, and educating physicians.

This database could be queried to identify high-volume diagnoses and treatments. These data were discussed by the utilization management committee of the medical group to determine how the group would manage its risk contract, including when educational interventions were needed. The medical group developed its own drug utilization reports, which were created using larger drug categories (e.g., First DataBank generic code number [GCN]) to better aggregate the data to support assimilation by physician-prescribers. The monthly utilization reports were reviewed by the Utilization Management Committee, a multidisciplinary committee made up of medical staff representatives, the director of case management, the clinical pharmacist, the chief financial officer, and the executive director; each region was represented by a physician.

The reports of the committee were disseminated electronically to allow peer review by the medical staff. The reports also provided feedback on the use of the recommended treatments in the 8 drug classes. (Therapeutic categories were rotated each month until all 8 target drug classes had been examined.) Physician members of the committee discussed the reports with their peers at weekly regional meetings. Since group meetings were conducted quarterly and medical service meetings were conducted bimonthly, the regional meetings permitted faster dissemination of the information.

To educate the medical staff on new medical information, concise therapeutic updates and new drug reviews were e-mailed weekly. The medical group had high participation in the e-mail network, which facilitated rapid and efficient dissemination of information.

To make the prescribing recommendations easily accessible, a quick-reference card was developed and distributed to the medical staff, physician extenders, nursing staff, and medical 


\begin{tabular}{|c|c|c|c|c|c|c|c|c|c|c|}
\hline & \multicolumn{5}{|c|}{ Novartis Pharmacy Benefit Report Data ${ }^{1,2}$} & \multicolumn{5}{|c|}{ Medical Group } \\
\hline & \multicolumn{2}{|c|}{1998} & \multicolumn{2}{|c|}{1999} & & \multicolumn{2}{|c|}{1998} & \multicolumn{2}{|c|}{1999} & \multirow[b]{2}{*}{$\%$ Change } \\
\hline & $\begin{array}{c}\text { PMPY } \\
\text { Cost }(\$)\end{array}$ & $\%$ Total & $\begin{array}{c}\text { PMPY } \\
\text { Cost }(\$)\end{array}$ & $\%$ Total & $\%$ Change & $\begin{array}{c}\text { PMPY } \\
\text { Cost }(\$)\end{array}$ & $\%$ Total & $\begin{array}{c}\text { PMPY } \\
\text { Cost }(\$)\end{array}$ & $\%$ Total & \\
\hline$\overline{\text { Antidepressants }}$ & 20.85 & 10.1 & 25.95 & $9.6 \%$ & 24.5 & 28.55 & 11.7 & 27.56 & 11.1 & -3.5 \\
\hline Statins & 16.64 & 8.0 & 23.17 & $8.5 \%$ & $39 . .2$ & 18.08 & 7.4 & 19.89 & 8.0 & 10.0 \\
\hline Antibiotics & 17.80 & 8.6 & 20.95 & $7.7 \%$ & 17.7 & 19.38 & 7.9 & 15.09 & 6.1 & -22.1 \\
\hline$\overline{\text { PPIs }}$ & 13.02 & 6.3 & 17.56 & $6.5 \%$ & 34.9 & 12.34 & 5.0 & 13.59 & 5.5 & 10.1 \\
\hline$\overline{\mathrm{H}_{2} \mathrm{~S}}$ & 6.66 & 3.2 & 4.41 & $1.6 \%$ & -33.8 & 4.49 & 1.8 & 3.38 & 1.4 & -24.7 \\
\hline$\overline{\mathrm{CCBS}}$ & 10.15 & 4.9 & 12.06 & $4.4 \%$ & 18.8 & 4.96 & 2.0 & 5.21 & 2.1 & 5.0 \\
\hline$\overline{\text { NSAIDs }}$ & 2.55 & 1.2 & 6.82 & $2.5 \%$ & 167.5 & 6.59 & 2.7 & 6.46 & 2.6 & -2.0 \\
\hline$\overline{\text { ACEIs }}$ & 8.83 & 4.3 & 10.41 & $3.8 \%$ & 17.9 & 6.21 & 2.5 & 5.28 & 2.1 & -15.0 \\
\hline Antihistamines & 8.33 & 4.0 & 10.51 & $3.9 \%$ & 26.2 & 14.63 & 6.0 & 12.15 & 4.9 & -17.0 \\
\hline NSs & 3.59 & 1.7 & 4.56 & $1.7 \%$ & 27.0 & 5.71 & 2.3 & 4.48 & 1.8 & -21.5 \\
\hline Other & 98.34 & 47.6 & 134.92 & $49.7 \%$ & 37.2 & 124.12 & 50.6 & 136.13 & 55.6 & 9.7 \\
\hline All drugs & 206.76 & 100.0 & 271.32 & 100.0 & 31.2 & 245.06 & 100.0 & 249.22 & 100.0 & 1.7 \\
\hline \multicolumn{11}{|c|}{$\begin{array}{l}\text { ACEIs = angiotensin-converting enzyme inhibitors: captopril, moexipril, trandolapril, fosinopril, benazepril, quinapril, ramipril, lisinopril, enalapril. } \\
\text { Antibiotics = amoxicillin, amoxicillin/clavulanate, azithromycin, cefaclor, cefdinir, cefixime, cefpodoxime, cefprozil, cefuroxime, cephalexin, cephradine, cefadroxil, } \\
\text { ciprofloxacin, clarithromycin, clindamycin, dicloxacillin, doxycycline, erythromycin, levofloxacin, loracarbef, metronidazole, nitrofurantoin, norfloxacin, ofloxacin, } \\
\text { penicillin V, trimethoprim-sulfimethoxazole, trimethoprim, trovafloxacin. }\end{array}$} \\
\hline \multicolumn{11}{|c|}{$\begin{array}{l}\text { Antidepressants = citalopram, fluvoxamine, paroxetine, fluoxetine, sertraline, venlafaxine, bupropion, mirtazapine, nefazodone, amitriptyline, doxepin, imipramine, } \\
\text { proptriptyline, desipramine, nortriptyline, trazodone. }\end{array}$} \\
\hline \multirow{2}{*}{\multicolumn{11}{|c|}{$\begin{array}{l}\text { Antihistamines = brompheniramine, cetirizine, fexofenidine, loratadine, and all combinations with pseudoephedrine. } \\
C C B s=\text { calcium channel blockers: amlodipine, felodipine, isradipine, nicardipine, nislodipine. }\end{array}$}} \\
\hline & & & & & lipine. & & & & & \\
\hline \multicolumn{11}{|c|}{$\mathrm{H}_{2} \mathrm{~S}=$ histamine-2 receptor blockers: cimetidine, ranitidine, nizatidine, famotidine. } \\
\hline \multicolumn{11}{|c|}{ NSs = nasal steroids: beclomethasone, budesonide, flunisolide, fluticasone, mometasone, triamcinolone. } \\
\hline \multicolumn{11}{|c|}{$\begin{array}{l}\text { NSAIDs = nonsteroidal anti-inflammatory drugs: celecoxib, diclofenac, diclofenac/misoprostol, etodolac, fenprofen, flurbiprofen, ibuprofen, indomethacin, ketoprofen, } \\
\text { ketorolac, nabumetone, naproxen, oxaprozin, piroxicam, rofecoxib, sulindac, tolmetin. }\end{array}$} \\
\hline \multicolumn{11}{|c|}{$\begin{array}{l}\text { PMPY = per member per year. The medical group had 12,128 members in } 1998 \text { and 11,119 members in } 1999 . \\
\text { PPIs = proton pump inhibitors: omeprazole, lansoprazole. }\end{array}$} \\
\hline \multicolumn{11}{|c|}{$\begin{array}{l}\text { PPIs = proton pump inhibitors: omeprazole, lansoprazole. } \\
R x=\text { prescription drug. }\end{array}$} \\
\hline \multicolumn{11}{|c|}{$R x=$ prescription drug. } \\
\hline & & & & & & & & & & \\
\hline
\end{tabular}

assistants. This reference card was a 1-sided page with information on all 8 drug therapy classes. A pocket-sized reference that included prices and formulary status for 4 different health plans was developed and distributed to physicians. This allowed a quick comparison of treatment alternatives. To create a more personal approach, the clinical pharmacist also reviewed drug utilization at monthly meetings and through medical office visits to physicians individually and collectively at a practice site.

\section{Data Analyses}

The terms of the global risk contract between the medical group and the health plan specified that all prescriptions were charged against the drug risk pool as the average wholesale price (AWP) of the drug minus the member copayment. Use of AWP minus the copayment inflated the base costs above the actual costs incurred by the HMO since pharmacy provider discounts and rebates received by the HMO and PBM from drug manufacturers were not shared with the medical group. The medical group was responsible for $25 \%$ of the deficit of the drug risk pool when the expenditures were more than the budgeted amount. If the drug expenditures were less than budgeted, the group would receive $75 \%$ of surplus. In the Novartis reports, drug cost was defined as actual ingredient cost of dispensed prescriptions, not including discounts, rebates, pharmacy fees, or copayments. Therefore, there is not a common definition of drug cost between the medical group and the national data, and percentage changes in therapeutic categories as they pertain to the total drug budget and percentage changes in utilization may provide a better comparison than absolute PMPY cost measures.

The utilization changes for total PMPY cost and PMPY claims volume for 8 drug classes and overall drug use were compared for the group data and national averages for the years 1998 and 1999. The percentage of the total utilization for each drug class was calculated to determine differences in the medical group's drug use compared with the national distribution by drug class. The average cost per claim for each therapeutic area was also compared. Since total drug expenditure is a product of item (prescription) cost and volume (prescriptions dispensed), 
a much lower average cost per claim could overcome an increase in prescription volume and thereby reduce the rate of increase in drug benefit expenditures. Focusing on the average cost per claim also permitted a clearer assessment of the magnitude of effect that each recommended (preferred) drug had on the drug class, the average cost for all prescription drug claims, and the entire drug budget.

\section{Results}

The PMPY expenditures for drugs increased $31.2 \%$ nationally for all health plan types, while this medical group limited the increase in its drug expenditures to $1.7 \%$ for its risk contract for commercial health plan members. (Table 1) PMPY drug costs in the medical group decreased in 5 of the 8 therapeutic classes and increased $5 \%$ to $10.1 \%$ in each of 3 drug classes: proton pump inhibitors (PPIs) (10.1\%), statins (10\%), and CCBs (5\%). However the increase in these 3 therapeutic areas was one third of the total PMPY cost increase in the national data.

The average cost per prescription claim increased by $31.2 \%$ in the national data, while the average cost per claim in the medical group decreased by $2.1 \%$ (Table 2 ). The average cost per prescription claim in the medical group decreased in all therapeutic areas except PPIs, where the cost remained the same in 1999 compared with 1998. The medical group performance in reducing the average cost per claim was superior to the national benchmark costs in every drug class except $\mathrm{H}_{2}$-blockers where the percentage changes were similar.

Nationally, PMPY prescription utilization did not change, while drug utilization in the medical group increased $4 \%$ (Table 3). The result of the clinical pharmacist interventions by therapeutic area were:

Allergy. While it was expected that use of nasal corticosteroids would increase and use of antihistamines would decrease, PMPY utilization of both drug classes was unchanged in 1999 compared with 1998 in the medical group. However, the desired outcome of a lower cost per prescription claim was achieved in both drug classes, $-20.4 \%$ for nasal steroids and $-15.4 \%$ for antihistamines.(Table 2), contributing to PMPY reductions of $21.5 \%$ for nasal steroids and $17 \%$ for antihistamines (Table 1).

Nonulceratine dyspepsia and gastroesophageal reflux disease. It was expected that both PPIs and $\mathrm{H}_{2}$-blockers would have a modest increase in utilization. The combined utilization of PPIs and $\mathrm{H}_{2}$-blockers increased by $9.1 \%$ (0.22 prescriptions PMPY to 0.24 prescriptions PMPY) in the medical group compared with $11.5 \%$ (0.26 prescriptions PMPY to 0.29 prescriptions PMPY) in the national data (Table 3). The increase in utilization of $\mathrm{H}_{2}$-blockers offset only one third of the $31.6 \%$ reduction in the average cost per claim for $\mathrm{H}_{2}$-blockers in the medical group, resulting in a $24.7 \%$ decrease in PMPY cost for $\mathrm{H}_{2}$-blockers. The combined cost for $\mathrm{H}_{2}$-blockers and PPIs, used primarily to treat heartburn and esophageal reflux disease, was
TABLE 2 Average Cost per Prescription Drug Claim

\begin{tabular}{|c|c|c|c|c|c|c|}
\hline & \multicolumn{3}{|c|}{$\begin{array}{l}\text { Novartis Pharmacy } \\
\text { Benefit Report Data }\end{array}$} & \multicolumn{3}{|c|}{ Medical Group } \\
\hline & $\begin{array}{l}1998 \\
(\$)\end{array}$ & $\begin{array}{c}1999 \\
(\$)\end{array}$ & $\begin{array}{c}\% \\
\text { Change }\end{array}$ & $\begin{array}{c}1998 \\
(\$)\end{array}$ & $\begin{array}{c}1999 \\
(\$)\end{array}$ & $\begin{array}{c}\% \\
\text { Change }\end{array}$ \\
\hline Antidepressants & 53.46 & 56.41 & 5.5 & 52.79 & 52.25 & -1.0 \\
\hline Statins & 64.00 & 64.36 & 0.6 & 71.90 & 68.80 & -4.3 \\
\hline Antibiotics & 25.43 & 26.86 & 5.6 & 16.46 & 14.04 & -14.7 \\
\hline PPIs & 108.50 & 117.07 & 7.9 & 106.82 & 106.99 & 0.2 \\
\hline$\overline{\mathrm{H}_{2} \mathrm{~S}}$ & 47.57 & 31.50 & -33.8 & 44.46 & 30.39 & -31.6 \\
\hline$\overline{\mathrm{CCBS}}$ & 40.60 & 38.90 & -4.2 & 47.64 & 43.84 & -8.0 \\
\hline NSAIDs & 19.62 & 22.00 & 12.2 & 19.92 & 18.62 & -6.5 \\
\hline$\overline{\text { ACEIs }}$ & 27.38 & 27.39 & -0.7 & 26.91 & 19.99 & -25.7 \\
\hline Antihistamines & 46.28 & 50.05 & 8.1 & 49.66 & 42.03 & -15.4 \\
\hline NSs & 35.90 & 35.08 & -2.3 & 33.46 & 26.64 & -20.4 \\
\hline Other & 16.93 & 26.10 & 54.2 & 23.46 & 24.40 & 4.0 \\
\hline All drugs & 24.61 & 32.30 & 31.2 & 28.55 & 27.95 & -2.1 \\
\hline
\end{tabular}

ACEIs = angiotensin-converting enzyme inhibitors: captopril, moexipril, trandolapril, fosinopril, benazepril, quinapril, ramipril, lisinopril, enalapril.

Antibiotics = amoxicillin, amoxicillin/clavulanate, azithromycin, cefaclor, cefdinir, cefixime, cefpodoxime, cefprozil, cefuroxime, cephalexin, cephradine, cefadroxil ciprofloxacin, clarithromycin, clindamycin, dicloxacillin, doxycycline, erythromycin, levofloxacin, loracarbef, metronidazole, nitrofurantoin, norfloxacin, ofloxacin, penicillin $V$, trimethoprim-sulfimethoxazole, trimethoprim, trovafloxacin.

Antidepressants = citalopram, fluvoxamine, paroxetine, fluoxetine, sertraline, venlafaxine, bupropion, mirtazapine, nefazodone, amitriptyline, doxepin, imipramine, proptriptyline, desipramine, nortriptyline, trazodone.

Antihistamines = brompheniramine, cetirizine, fexofenidine, loratadine, and all combinations with pseudoephedrine.

$C C B s$ = calcium channel blockers: amlodipine, felodipine, isradipine, nicardipine, nislodipine.

$\mathrm{H}_{2} \mathrm{~S}=$ histamine-2 receptor blockers: cimetidine, ranitidine, nizatidine, famotidine.

NSs = nasal steroids: beclomethasone, budesonide, flunisolide, fluticasone, mometasone, triamcinolone.

NSAIDs = nonsteroidal anti-inflammatory drugs: celecoxib, diclofenac, diclofenacl misoprostol, etodolac, fenprofen, flurbiprofen, ibuprofen, indomethacin, ketoprofen, ketorolac, nabumetone, naproxen, oxaprozin, piroxicam, rofecoxib, sulindac, tolmetin.

$P M P Y=$ per member per year. The medical group had 12,128 members in 1998 and 11,119 members in 1999.

PPIs = proton pump inhibitors: omeprazole, lansoprazole

$R x=$ prescription drug.

Statins = cerivastatin, fluvastatin, atorvastatin, lovastatin, pravastatin, simvastatin.

essentially unchanged at \$16.97 PMPY in 1999 compared with $\$ 16.83$ in 1998. This favorable cost outcome compared with an $11.6 \%$ increase in combined PMPY $\mathrm{H}_{2}$-blockers and PPI costs in the national data (\$19.68 in 1998 and \$21.97 in 1999); the medical group costs were $23 \%$ lower than the national data in 1999 ( $\$ 16.87$ PMPY versus \$21.97 PMPY) (Table 1). The large reduction in the average cost per claim for $\mathrm{H}_{2}$-blockers was due primarily to greater use of generic ranitidine.

Depression. The utilization of antidepressants in the medical group was little changed at 0.54 prescriptions PMPY in 1998 and 0.53 prescriptions PMPY in 1999) compared with a $17.9 \%$ increase ( 0.39 prescriptions PMPY to 0.46 prescriptions PMPY) in the national data (Table 3). The small decease in utilization of antidepressants may have been associated with a review of the appropriateness of drug continuation in each patient. There was a $1 \%$ decrease in the average cost per claim 
TABLE 3 Utilization (Drug Claim Volume) for Medical Group Compared With National Data

\begin{tabular}{|c|c|c|c|c|c|c|c|c|c|c|}
\hline & \multicolumn{5}{|c|}{ Novartis Pharmacy Benefit Report Data ${ }^{1,2}$} & \multicolumn{5}{|c|}{ Medical Group } \\
\hline & \multicolumn{2}{|c|}{1998} & \multicolumn{2}{|c|}{1999} & & \multicolumn{2}{|c|}{1998} & \multicolumn{2}{|c|}{1999} & \\
\hline & $\begin{array}{l}\text { Rx Claims } \\
\text { PMPY }\end{array}$ & $\%$ Total & $\begin{array}{c}\text { Rx Claims } \\
\text { PMPY }\end{array}$ & $\%$ Total & $\%$ Change & $\begin{array}{l}\text { Rx Claims } \\
\text { PMPY }\end{array}$ & $\%$ Total & $\begin{array}{l}\text { Rx Claims } \\
\text { PMPY }\end{array}$ & $\%$ Total & $\%$ Change \\
\hline Antidepressants & 0.39 & 4.6 & 0.46 & 6.4 & 17.9 & 0.54 & 6.3 & 0.53 & 5.9 & -1.9 \\
\hline Statins & 0.26 & 3.1 & 0.36 & 5.0 & 38.5 & 0.25 & 2.9 & 0.29 & 3.3 & 16.0 \\
\hline Antibiotics & 0.70 & 8.3 & 0.78 & 10.8 & 11.4 & 1.17 & 13.6 & 1.08 & 12.1 & -8.1 \\
\hline$\overline{\text { PPIs }}$ & 0.12 & 1.4 & 0.15 & 2.1 & 25.0 & 0.12 & 1.4 & 0.13 & 1.4 & 9.5 \\
\hline$\overline{\mathrm{H}_{2} \mathrm{~S}}$ & 0.14 & 1.7 & 0.14 & 1.9 & 0 & 0.10 & 1.2 & 0.11 & 1.3 & 10.9 \\
\hline$\overline{\mathrm{CCBS}}$ & 0.25 & 3.0 & 0.31 & 4.3 & 24.0 & 0.10 & 1.2 & 0.12 & 1.3 & 20.0 \\
\hline$\overline{\text { NSAIDs }}$ & 0.13 & 1.5 & 0.31 & 3.7 & 138.5 & 0.33 & 3.9 & 0.35 & 3.9 & 4.8 \\
\hline$\overline{\text { ACEIs }}$ & 0.32 & 3.8 & 0.38 & 5.3 & 18.8 & 0.21 & 2.4 & 0.26 & 2.9 & 23.8 \\
\hline Antihistamines & 0.18 & 2.1 & 0.21 & 2.9 & 16.7 & 0.29 & 3.4 & 0.29 & 3.3 & $\overline{0}$ \\
\hline$\overline{\mathrm{NSs}}$ & 0.10 & 1.2 & 0.13 & 1.8 & 30.0 & 0.17 & 2.0 & 0.17 & 1.9 & 0 \\
\hline Other & 5.81 & 69.2 & 5.17 & 61.5 & -11.0 & 5.29 & 61.7 & 5.58 & 62.6 & 5.5 \\
\hline All drugs & 8.40 & 100.0 & 8.40 & 100.0 & 0 & 8.57 & 100.0 & 8.91 & 100.0 & 4.0 \\
\hline
\end{tabular}

ACEIs = angiotensin-converting enzyme inhibitors: captopril, moexipril, trandolapril, fosinopril, benazepril, quinapril, ramipril, lisinopril, enalapril.

Antibiotics = amoxicillin, amoxicillin/clavulanate, azithromycin, cefaclor, cefdinir, cefixime, cefpodoxime, cefprozil, cefuroxime, cephalexin, cephradine, cefadroxil,

ciprofloxacin, clarithromycin, clindamycin, dicloxacillin, doxycycline, erythromycin, levofloxacin, loracarbef, metronidazole, nitrofurantoin, norfloxacin, ofloxacin, penicillin V, trimethoprim-sulfimethoxazole, trimethoprim, trovafloxacin.

Antidepressants = citalopram, fluvoxamine, paroxetine, fluoxetine, sertraline, venlafaxine, bupropion, mirtazapine, nefazodone, amitriptyline, doxepin, imipramine, proptriptyline, desipramine, nortriptyline, trazodone.

Antihistamines = brompheniramine, cetirizine, fexofenidine, loratadine, and all combinations with pseudoephedrine.

$C C B s=$ calcium channel blockers: amlodipine, felodipine, isradipine, nicardipine, nislodipine.

$\mathrm{H}_{2} \mathrm{~S}=$ histamine-2 receptor blockers: cimetidine, ranitidine, nizatidine, famotidine.

NSs = nasal steroids: beclomethasone, budesonide, flunisolide, fluticasone, mometasone, triamcinolone

NSAIDs = nonsteroidal anti-inflammatory drugs: celecoxib, diclofenac, diclofenac/misoprostol, etodolac, fenprofen, flurbiprofen, ibuprofen, indomethacin, ketoprofen,

ketorolac, nabumetone, naproxen, oxaprozin, piroxicam, rofecoxib, sulindac, tolmetin

$P M P Y=$ per member per year. The medical group had 12,128 members in 1998 and 11,119 members in 1999.

PPIs = proton-pump inhibitors: omeprazole, lansoprazole.

$R x=$ prescription drug.

Statins = cerivastatin, fluvastatin, atorvastatin, lovastatin, pravastatin, simvastatin

for antidepressants (\$52.79 in 1998 and \$52.25 in 1999) in the medical group compared with a $5.5 \%$ increase in the national data. The medical group experienced a 3.5\% reduction in PMPY costs for antidepressants in 1999 compared with a 24.5\% increase in the national data. The medical group costs were $6 \%$ higher than the national data in 1999 (\$27.56 PMPY versus $\$ 25.95$ PMPY) due to higher utilization in the medical group compared with the national data (Table 1).

Antibiotics. It was expected that utilization of antibiotics would fall as patients and physicians were educated in the use of antibiotics. Antibiotic utilization decreased by $8.1 \%$ (1.17 prescriptions PMPY to 1.08 prescriptions PMPY) in the medical group compared with an $11.4 \%$ increase ( 0.70 prescriptions PMPY to 0.78 prescriptions PMPY) in the national data (Table 3). The average cost per claim decreased 14.7\% (\$16.46 per claim to $\$ 14.04$ per claim) compared with a $5.6 \%$ increase in the cost per claim ( $\$ 25.43$ per claim to $\$ 26.86$ per claim) in the national data. The decrease in antibiotic utilization and average cost per claim resulted in a $22.1 \%$ decrease in PMPY costs
(\$19.38 in 1998 and \$15.09 in 1999 in the medical group). This positive cost outcome compared with a 17.7\% increase in PMPY antibiotic costs in the national data (\$17.80 in 1998 and $\$ 20.95$ in 1999), and the medical group costs were $28 \%$ lower than the national data in 1999 (\$15.09 PMPY versus \$20.95 PMPY) (Table 1). The decrease in the cost per claim for antibiotics resulted from an increase in the use of generic first-line antibiotics.

Angiotensin-converting enzyme inhibitors (ACEIs). The utilization of ACEIs increased 23.8\% (0.21 prescriptions PMPY to 0.26 prescriptions PMPY) in the medical group, similar to the $18.8 \%$ increase (0.32 prescriptions PMPY to 0.38 prescriptions PMPY) in the national data (Table 3 ). There was a $25.7 \%$ reduction in the average cost per claim (\$26.91 in 1998 to \$19.99 in 1999) in the medical group compared with essentially no change in the national data (\$27.38 in 1998 to \$27.39 in 1999) (Table 2). The reduction in the average cost per claim overwhelmed the increase in utilization, resulting in a 15\% decrease in PMPY costs (\$6.21 in 1998 and \$5.28 in 1999 in the medical group). This compared favorably with a $17.9 \%$ increase in PMPY 
costs in the national data (\$8.83 in 1998 and $\$ 10.41$ in 1999). The medical group's costs were $49.4 \%$ lower than the national data in 1999 (\$5.28 PMPY versus \$10.41 PMPY) (Table 1).

Calcium channel blockers (CCBs). The utilization of CCBs increased 20\% (0.10 prescriptions PMPY to 0.12 prescriptions PMPY) in the medical group compared with 24\% (0.25 prescriptions PMPY to 0.31 prescriptions PMPY) in the national data (Table 3). There was an $8 \%$ reduction in the average cost per claim (\$47.64 in 1998 to $\$ 43.84$ in 1999) compared with a $4.2 \%$ decrease in the national data ( $\$ 40.60$ in 1998 to $\$ 38.90$ in 1999) (Table 2). The increase in utilization of CCBs offset the reduction in the average cost per claim, resulting in a 5\% increase in PMPY costs (\$4.96 in 1998 and \$5.21 in 1999). This compared favorably with an $18.9 \%$ increase in PMPY costs in the national data (\$10.15 in 1998 and \$12.06 in 1999), and the medical group's costs were $56.8 \%$ lower than the national data in 1999 (\$5.21 PMPY versus \$12.06 PMPY) (Table 1).

Dyslipidemia and statin therapy. The utilization of statins increased 16\% (0.25 prescriptions PMPY to 0.29 prescriptions PMPY) in the medical group compared with 37\% (0.26 prescriptions PMPY to 0.36 prescriptions PMPY) in the national data (Table 3). There was a $4.3 \%$ reduction in the average cost per statin claim (\$71.90 in 1998 to $\$ 68.80$ in 1999) compared with a $0.6 \%$ increase in the national data (\$64.00 in 1998 to $\$ 64.36$ in 1999) (Table 2). The $16 \%$ increase in utilization of statins overwhelmed the $4.3 \%$ reduction in the average cost per statin claim in the medical group to cause a 10\% increase in PMPY costs (\$18.08 in 1998 and $\$ 19.89$ in 1999). This cost outcome was favorable, however, compared with the 39\% increase in PMPY costs in the national data (\$16.64 in 1998 and $\$ 23.17$ in 1999), and the medical group's costs were $14.2 \%$ lower than the national data in 1999 ( $\$ 19.89$ PMPY versus $\$ 23.17$ PMPY) (Table 1). Use of statins increased, but not as much as statin use did nationally. Examination of the prevention of future coronary events through the use of aggressive cholesterol lowering was defined as an important target for future quality improvement interventions. The reduction in the average cost per statin prescription in 1999 offset the increase in utilization to produce a smaller increase in the PMPY statin cost.

Nonsteroidal anti-inflammatory drugs (NSAIDs). Combined NSAID and COX-2 utilization increased by $4.8 \%$ (0.33 prescriptions PMPY to 0.35 prescriptions PMPY) in the medical group compared with a $138.5 \%$ increase in the combined NSAID and COX-2 national utilization $(0.13$ prescriptions PMPY to 0.31 prescriptions PMPY) (Table 3). The average cost per claim decreased $6.5 \%$ ( $\$ 19.92$ per claim to $\$ 18.62$ per claim) compared with a $12.2 \%$ increase in the cost per claim ( $\$ 19.62$ per claim to $\$ 22.00$ per claim) in the national data. A greater use of generic NSAIDs and low use of COX-2s led to a lower cost per claim in the medical group. The decrease in the average cost per claim offset the increase utilization and resulted in a 2\% decrease in PMPY costs (\$6.59 in 1998 and
$\$ 6.46$ in 1999). This positive cost outcome compared with a $167.5 \%$ increase in PMPY costs in the national data $(\$ 2.55$ in 1998 and $\$ 6.82$ in 1999) (Table 1).

Generic drug claims in the medical group increased by 3 percentage points (6.3\% relative change) from $48 \%$ in 1998 to $51 \%$ in 1999 compared with a national average of $45.6 \%$ in 1998 an 47\% in 1999 (3.1\% relative change). The reduced rate of increase in drug expenditures in 1999 in this medical group was attributable primarily to increased utilization of preferred drugs and secondarily to increased utilization of generic drugs. While overall prescription claim volume increased $4.0 \%$ from 8.57 in 1998 to 8.91 prescriptions PMPY in 1999, the 2.1\% reduction in the average cost per claim (from $\$ 28.55$ in 1998 to $\$ 27.95$ in 1999), resulted in a $1.7 \%$ increase in PMPY cost (to $\$ 249.22$ in 1999 from $\$ 245.06$ in 1998).

\section{Discussion}

Changes in PMPY drug costs are influenced by changes in both average cost per drug claim and drug utilization. The average cost per prescription tends to increase over time due to manufacturer price increases and a change in the mix of drugs, such as the use of a PPI for heartburn rather than an $\mathrm{H}_{2}$-blocker; the cost per day of therapy in 1999 was at least 3 times higher for the PPI compared with the $\mathrm{H}_{2}$-blocker. The introductory material presented in a recent study sponsored by the pharmaceutical industry concluded that increased utilization was the major cost driver to higher drug expenditures. ${ }^{7}$ However, 2 other research groups have found that the increase in drug expenditures is influenced more by an increase in drug price (the 2002 report produced by the National Institute of Health Care Management, sponsored by BlueCross and BlueShield health plans, and a 2002 report from Express Scripts). 8,9

When a brand drug loses patent protection, a lower-priced generic alternative becomes available, creating the opportunity for a cost reduction for the therapeutic class as well as the specific drug. Conversely, the market introduction of a new, higher-cost drug can increase the direct drug cost for treating the disease-indication as prescription utilization migrates toward the new drug. Other health care and drug cost drivers include an aging population and the use of diagnostic tests for disease screening and monitoring. Increased awareness of a disease (e.g., depression) often contributes to increased utilization of pharmacotherapy for the disease.

Many strategies have been used to modify physician prescribing behavior. Educational interventions have demonstrated success in changing physician prescribing habits. Academic detailing, also known as counter-detailing or educational outreach, has been shown to change physician practice and be cost effective. ${ }^{6}$ Pharmacist-initiated medication review followed by a recommendation has been shown to lower prescription cost and volume in a Medicare managed care population. ${ }^{10}$ An education program was shown to decrease the use of psycho- 
active drugs in a long-term care population. ${ }^{11}$ An intervention to decrease the use of inappropriate dipyridamole for cardiovascular disease prophylaxis found that more patients had their dipyridamole prescription discontinued when an educational letter was sent to physicians and pharmacists compared with patients who had a letter sent only to their physician or only to their pharmacist. ${ }^{12}$

Traditional continuing education programs and clinical guidelines have been shown to have minimal impact on changing physician practice habits. ${ }^{13}$ However, endorsement by local opinion leaders combined with structured feedback increases the acceptance of preventative practice guidelines for common diseases such as hypertension, chronic obstructive lung disease, and colorectal cancer screening. ${ }^{14}$ Greater acceptance of a preferred drug list (PDL) can be achieved by tailoring the message of a targeted newsletter. ${ }^{15}$ Use of restrictive formularies ${ }^{16}$ has been shown to decrease the use of nonformulary drugs, but the cost outcomes of this shift in therapy depends upon the content of the drug formulary (the PDL).

A systematic review of 18 studies involving more than 1,896 physicians examined the impact of outreach visit interventions. An outreach visit was defined as an intervention that has the potential to change health professional practice, particularly prescribing by physicians. The interventions included social marketing methods, printed information, and personal conferences. Social marketing is the application of communication and behavioral change principles to persuade an individual to make a change, e.g., positive reinforcement, using opinion leaders and encouraging participation. These methods have been successfully employed by the pharmaceutical industry for many years. Participant feedback was used in some of the interventions.

Thirteen of the studies involved modifying prescribing behavior. All studies demonstrated a desired effect. The authors concluded that educational outreach visits, particularly when combined with social marketing, appear to be a promising approach to modifying behavior, especially prescribing. It was noted that, while outreach visits are effective, the cost-effectiveness of outreach visits has not been well evaluated. ${ }^{17}$

Interventions at the point of care also provide a means to affect behavioral change. Making information on drug cost available to physicians through physician order-entry systems has demonstrated success in decreasing drug costs..$^{18,19}$ Use of preformatted chart notes can change physician practice habits. This strategy is especially useful in the nonacademic setting where structured educational rounds are not available. ${ }^{20}$

Abourjaily, Kross, and Gouveia reported that pharmacistmediated interventions were successful in limiting growth in drug expenditures in an independent physician association with a drug risk contract. These interventions included educating providers on drug selection, creating a reference for preferred drugs based on analysis of drug utilization, cus- tomized utilization reports, and quality control reviews. These initiatives allowed the group to reduce the increase in drug expenditures to less than the national average..$^{21}$ These methods were very similar to the ones used by Esse Health in this study. We found that a quick review list of preferred drugs, customized utilization reports, peer pressure, and use of idea champions, was effective in reducing the rate of growth of drug expenditures PMPY in our medical group.

The interventions by Abourjaily, Kross, and Gouveia resulted in an average annual increase in per-member-per-month (PMPM) drug expenditures of 10\% or less from 1997 to 2000. The only exception was 1999 when PMPM drug expenditures increased by $14.7 \%$. At Esse Health, PMPY (which could be expressed as PMPM), drug expenditures increased only $1.7 \%$ in 1999. Since the program only lasted 1 year, it is not possible to predict what future drug expenditures would have been. In 2000, Abourjaily, Kross, and Gouveia reported their drug expenditures to be $11 \%$ under budget. In 1999, the drug expenditures at Esse Health were 13\% under budget. The absolute dollar amounts of the PMPM (or PMPY) budgets for the 2 groups are not comparable due to different definitions of drug cost. However, results of the 2 interventions suggest that both managed care organizations would have been conservative in setting the budget for the groups. This provides some support for the influence of a clinical pharmacy service in controlling drug expenditures in a large physician group.

When selecting the educational interventions for the medical group in this study, the unique dynamics of the group and the nonacademic community setting were factors that were considered. The availability of active e-mail discussions, a multidisciplinary utilization committee, and regional medical staff meetings provided an infrastructure for dissemination of educational information.

Participation by the pharmacist in the meetings and discussions provided a means to gain credibility and encourage physician participation. It also provided a method to determine the group leaders and opinion makers. By using the group leaders and opinion makers as idea champions, proposed prescribing changes were also seen as being physician-to-physician and not just pharmacist-to-physician. Succinct reviews of treatment strategies and new drugs provided a means to reinforce the desired practice changes. Use of reports to the medical staff provided feedback to encourage acceptance of the new prescribing patterns.

At the end of 1999, the managed care organization that held the global risk contract with the medical group changed its approach to managed care and eliminated the requirement for primary care physician preauthorization of visits to specialists. This essentially ended the role of primary care physicians as gatekeepers and thus ended the global risk contract. The savings to the drug risk pool became a part of the negotiation in the subsequent financial agreement.

(Article continues on page 353.) 


\section{Limitations}

We did not measure any clinical outcomes associated with these changes in drug utilization and reduction in the average price (cost per prescription). We therefore could not assess the effects of our drug cost and utilization outcomes on total medical care outcomes. However, 4 times during the year, the medical staff was informally queried as to whether they felt there was any change in office visits or patient satisfaction because of these prescription drug interventions. Each time, the staff indicated that they perceived no change in office visit volume or patient satisfaction. This suggests that service-humanistic outcomes were not adversely affected by this drug cost reduction program.

Use of national data for comparative purposes was useful but less desirable than cost and utilization data obtained from a control (medical) group operating without clinical pharmacist intervention. The national data source also used different metrics in the 2 measurement years. Another potential limitation, perhaps small, was the different calendar periods used for data collection in the Novartis reports, October 1 to September 30, while the medical group collected data in a calendar year. We also did not calculate or have access to summary demographic information for the managed care organization members assigned to the medical group. It is therefore not possible to either extrapolate our findings to other groups of MCO members or to riskadjust our own enrolled population, which declined in total numbers by $13.4 \%$ (from 14,005 to 12,218 ) in 1998 and by an additional 8.3\% in 1999 (from 12,218 to 11,119).

We also did not have the ability to use a control group of members within this medical group due to the open communication and fairly advanced degree of electronic networking among practicing physicians. The absence of a control group limits our ability to attribute precisely the observed change in average cost per prescription to the clinical pharmacist intervention. However, other potential confounders such as a change in drug benefit design appear to be minimized. The observed reduction in the average cost per prescription occurred despite the conclusion of the drug-risk contract period 2 months prior to the end of the study period.

Left to other researchers is a comparison of the relative effectiveness of a clinical pharmacist employed by the medical group compared with academic detailing performed by clinical pharmacists employed by the health plan or PBM. A number of other alternative intervention models are possible to permit daily interaction of a clinical pharmacist with prescribers to foster optimum working relationships and identify ways to tailor messages to individual prescribers and the needs of a given medical group.

\section{Conclusions}

The PMPY expenditures for drug therapy for health plan members assigned to this primary care medical group increased by only $1.7 \%$ after rising by $29.8 \%$ the previous year. The PMPY prescription claims volume increased by $4 \%$ overall, by $16 \%$ for statins, and by $20 \%$ or more for the 2 anithypertensive drug classes: ACE inhibitors (23.8\%) and calcium channel blockers (20.0\%). The increase in utilization of drugs for cardiovascular disease was offset by an $8.1 \%$ reduction in utilization of antibiotics. The average cost per claim for all drugs decreased by $2.1 \%$, unadjusted for inflation. The resultant suppression in drug benefit cost trend in the medical group was associated with the implementation and operation of a focused educational program for physician-prescribers. A clinical pharmacist armed with reliable, useful, and timely drug cost and utilization data that are physician-specific, concise therapeutic updates and who has access to physician-prescribers can have a favorable effect on the PMPY drug cost trend of a primary care medical group providing medical services under financial risk contract with a health plan.

\section{DISCLOSURES}

No outside funding supported this study. Authors Scot Walker and Charles W. Wiley disclose no conflict of interest or potential bias regarding this article. Walker served as principal author of the study and was responsible for drafting of the manuscript. Both authors contributed to study concept and design, analysis and interpretation of data, and the critical revision of the manuscript.

\section{REFERENCES}

1. Curtiss FR. Contractual arrangements between HMOs and medical groups to manage drug costs. J Manag Care Pharm. 2003;9(6):752-73.

2. Anonymous. Express Scripts 1999 Drug Trend Report. St. Louis, MO: Express Scripts; 2000.

3. Anonymous. Novartis pharmacy benefits report. Facts \& Figures. 1999 ed. East Hanover NJ: Novartis.

4. Anonymous. Novartis pharmacy benefits report. Facts \& Figures. 2000 ed. East Hanover NJ: Novartis.

5. Personal communication, Robin C. Emigh, ed. Novartis pharmacy benefits report. Facts \& Figures. August 5, 2003

6. Soumerai SB, Avorn J. Principles of educational outreach ("academic detailing") to improve clinical decision making. JAMA. 1990;263:549-56.

7. Gilberg K, Laouri M, Wade S, Isonaka, S. Analysis of medication use patterns: Apparent overuse of antibiotics and underuse of prescription drugs for asthma, depression, and CHF. J Manag Care Pharm. 2003;9(3):232-37.

8. National Institute for Health Care Management Research and Educational Foundation. Changing patterns of pharmaceutical innovation. Washington, DC; May 2002. Available at: www.nihcm.org. Accessed December 12, 2003.

9. Curtiss FR. Finding the truth about health care cost drivers-price versus utilization factors. J Manag Care Pharm. 2003;9(3):274-75

10. Bieszk N, Bhargava V, Pettita, Whitelaw N, Zarowitz B. Quality and cost outcomes of clinical pharmacist interventions in a capitated senior drug benefit. J Manag Care Pharm. 2002;8(2):124-31.

11. Avorn J, Soumerai SB, Everitt DE, et al. A randomized trial of a program to reduce the use of psychoactive drugs in nursing homes. N Engl J Med. 1992;327:168-73

12. Collins TM, Mott DA, Bigelow WE, Zimmerman DR. A controlled letter intervention to change prescribing behavior: the results of a dual-targeted approach. Health Serv Res. 1997;32:471-89.

13. Sbarbaro JA. Can we influence prescribing patterns. Clin Infect Dis. 2001;33(suppl 3):S240-S244. 
14. Lomas J, Haynes RB. A taxonomy and critical review of tested strategies for the application of clinical practice recommendations: from "official" to "individual" clinical policy. Am J Prev Med. 1988;4(suppl):77-94.

15. Walker SE, Martin WR. Focusing physician involvement in the formulary process. Pharm Times. 1996;62(1):6HPT-16HPT.

16. Dowell JS, Snadden D, Dunbar JA. Changing to generic formulary: how one fundholding practice reduced prescribing costs. BMJ. 1995;310:505-08.

17. O'Brien T, Oxman AD, Davis DA Haynes RB, Freemantle N, Harvey EL Educational outreach visits: effects on professional practice and health care outcomes. Cochrane Review. [The Cochrane Library]. 2002;4.
18. Ornstein SM, MacFarlane LL, Jenkins RG, Pan Q, Wager KA. Medication cost information in a computer-based patient record system. Impact on prescribing in a family medicine clinical practice. Arch Fam Med. 1999;8:118-21.

19. Vedsted P, Nielsen JN, Olesen F. Does a computerized price comparison module reduce prescribing costs in general practice? Fam Pract. 1997;14:199203.

20. Walker SE. Physicians acceptance of a pre-formatted pharmacy intervention chart note in a community hospital—antibiotic step-down program. J Pharm Technol. 1998;14:141-45.

21. Abourjaily P, Kross J, Gouveia WA. Initiatives to control drug costs associated with an independent physician association. Am J Health Syst Pharm. 2003;60:269-72. 